\title{
Aberrant Projection Induced by Otocyst Removal Maintains Normal Tonotopic Organization in the Chick Cochlear Nucleus
}

\author{
William R. Lippe, ${ }^{1,2}$ David S. Fuhrmann, ${ }^{1,2}$ Wenhua Yang, ${ }^{1}$ and Edwin W Rubel' \\ 'Department of Otolaryngology, University of Washington School of Medicine, Seattle, Washington 98195, and \\ ${ }^{2}$ Department of Veterans Affairs Medical Center, Seattle, Washington 98108
}

Nucleus magnocellularis (NM), a second-order nucleus in the chick auditory system, is topographically and tonotopically organized. The basilar papilla (cochlea) projects onto the ipsilateral NM via the auditory nerve. The anteromedial region of NM is innervated by the proximal end of the basilar papilla and responds to high-frequency sounds; more posterolateral regions receive input from more distal locations along the papilla and respond to progressively lower frequencies. NM projects exclusively to the third-order neurons of nucleus laminaris (NL). Otocyst removal prevents the formation of the ipsilateral cochlea and cochlear nerve and results in the development of an aberrant functional projectIon from the contralateral NM to the "deafferented" NM on the operated side of the brain (Jackson and Parks, 1988).

In the present experiment, the otocyst was removed unilaterally and the tonotopic organization of the deafferented NM was physiologically mapped at 17-18 d of embryonic age (E17-E18). Quantitative analyses revealed that the frequency organization of the deafferented NM is almost identical to that in normal embryos. Progressively higher characteristic frequencies were recorded at successively more anterior and more medial locations in the nucleus, and the orientation of the tonotopic axis was indistinguishable from normal. Furthermore, the correlation between characteristic frequency and anatomical location is comparable in the deafferented $(r=0.91)$ and normal $(r=0.87)$ NM. The only noticeable discrepancy is that characteristic frequencies in NM on both sides of the brain of operated embryos are higher than the frequencies observed previously at comparable regions of the nucleus in unoperated controls. This discrepancy most probably reflects a difference in developmental age between operated embryos (E17-E18) and the normal embryos to which they were compared (E16-E17) and is unrelated to the aberrant projection.

The observation that the induced NM-to-NM projection maintains a normal tonotopic organization in the deafferented NM implies that the projection is topographically organized. These findings also support the idea that NM is not a passive receiver of incoming afferents and that the ori-

\footnotetext{
Received June 11, 1991; revised Oct. 11, 1991; accepted Oct. 18, 1991.

We thank Karen Canady, Rick Hyson, and Tom Reh for their very helpful comments. We also thank an anonymous reviewer for the suggestion that the tonotopic map might result from preexisting fiber order. This work was supported by NIH Grants NS 20724 (W.R.L.) and DC 00395 (E.W R.) and by the Medical Research Service of the Department of Veterans Affairs (W.R.L.)

Correspondence should be addressed to Dr. William Lippe, Department of Otolaryngology, RL-30, University of Washington, Seattle, WA 98195.

Copyright (C) 1992 Society for Neuroscience $0270-6474 / 92 / 120962-08 \$ 05.00 / 0$
}

entation of the tonotopic axis in NM is determined by cues intrinsic to the nucleus. It is likely that the cues for orientation are similar in NM and NL, and that the induced NM branches are reading cues comparable to those which their parent axons normally encounter in their innervation of NL.

Receptor surfaces of the major sensory systems are represented topographically in the brain. The auditory nuclei have a tonotopic organization, the somatosensory system contains maps of the body surface, and nuclei in the visual system are organized retinotopically. These physiological maps are based on orderly patterns of anatomical connections. The sensory surfaces project topographically into the brain. Topography is preserved at all levels of the central sensory pathways by spatially ordered connections between sensory nuclei.

The question of how topographically ordered connections are set up during development has been a major focus of experimental neuroembryology and developmental neurobiology for the past 70 years. Much of our understanding has come from studies of abnormal axonal projections. These include anomalous projections that arise during the course of normal development as well as projections resulting from lesions, transplantation of sensory primordia, and other experimental manipulations (Guillery and Kaas, 1971; Law and ConstantinePaton, 1981; Pallas et al., 1990).

In normal avian embryos, nucleus magnocellularis (cochlear nucleus) receives a spatially ordered projection from the ipsilateral basilar papilla (cochlea) via the auditory nerve. The topography of this projection forms the basis for the tonotopic organization of nucleus magnocellularis (NM). Neurons at the anteromedial pole of NM are innervated by the proximal (highfrequency) end of the papilla and respond to high-frequency sounds; neurons at successively more posterolateral locations in the nucleus receive input from progressively more distal (lowfrequency) regions along the papilla and are tuned to progressively lower-frequency sounds (Boord and Rasmussen, 1963; Rubel and Parks, 1975; Parks and Rubel, 1978; Lippe and Rubel, 1985). NM, in turn, projects exclusively to nucleus laminaris (NL) bilaterally (Parks and Rubel, 1975; Young and Rubel, 1983, 1986). Extirpation of the otocyst in young embryos prevents the development of the basilar papilla and auditory nerve and induces the formation of a collateral axonal projection from the contralateral NM to the NM on the operated side of the brain (Fig. 1; Parks and Jackson, 1986). In vitro studies have shown that the abnormal projection establishes functional synaptic contacts with neurons in the deafferented NM (Jackson and Parks, 1988).

The purpose of the present study was to determine if the 
aberrant projection establishes a tonotopic organization in the deafferented NM and the degree to which this projection approaches the normal organization found in embryos of similar age (Lippe and Rubel, 1985; Lippe, 1987). The frequency organization of NM was mapped physiologically in 17-18-d-old embryos (E17-E18) that had undergone unilateral otocyst extirpation at 2-3 d of incubation. The results show that the deafferented NM is tonotopically organized, and that the organization is similar to that in the normal NM of unoperated embryos.

\section{Materials and Methods}

Subjects and otocyst removal. The right otocyst was surgically removed from approximately 450 White Leghorn chicken embryos following previously published procedures (Parks, 1979). Fertile eggs were incubated without turning for $3 \mathrm{~d}$ in a forced-draft incubator at $37.5^{\circ} \mathrm{C}$ and $50-60 \%$ relative humidity. A small hole was cut in the shell, and a drop of sterile $2 \%$ neutral red dye was placed on the membrane overlying the otocyst. The membrane was removed with a fine forceps, and the otocyst was pulled away with very finely sharpened tungsten needles. The hole in the shell was sealed with plastic tape, and the egg was returned to the incubator where it remained, without turning, until the day of recording.

Unoperated E16-E17 embryos from two prior experiments provided normative data on the tonotopic organization of NM (Lippe and Rubel, 1985; Lippe, 1987)

Preparation for recording. Embryos were prepared for electrophysiological recording after a total of $20 \mathrm{~d}$ of incubation. Procedures were similar to those used previously (Lippe and Rubel, 1985; Lippe, 1987). The egg was removed from the incubator, and the embryo's head was pulled through a small hole made in one end of the shell. The embryo was anesthetized with an injection into the dorsal neck muscle of ketamine hydrochloride (1.3 mg Ketalar; Parke-Davis/American Cyanamid). The region surrounding the ear was infiltrated with local anesthetic ( $1 \%$ lidocaine hydrochloride with $0.01 \%$ epinephrine; Elkins-Sin, Inc.). Spontaneous muscle movements were eliminated with gallamine triethiodide (1 mg Flaxedil, i.m.; American Cyanamid).

To obtain some indication of the success of otocyst removal prior to the physiological studics, the right middle ear was examincd by removing the tympanic membrane. We have observed that complete absence of the auditory nerve following otocyst removal is typically associated with abnormalities in middle ear structure, such as fusion of the columella to the tympanic cavity and occlusion of the oval and round windows. Therefore, any embryos whose right middle ear appeared normal were not studied electrophysiologically and were eliminated from the study.

The embryo was supported in a humidified and temperature-controlled chamber. The tissue, skull, and dura overlying the dorsal surface of the cerebellum were removed. Grass needle electrodes were inserted through two small holes in the shell in order to monitor the embryo's heart rate. Heart rate provided an indication of the physiological condition of the embryo throughout the experiment.

Sound presentation and calibration. The tissue surrounding the left ear was surgically removed in order to be able to visualize the ear canal and the tympanic membrane clearly. Sound was delivered to the left ear through a brass tube positioned over the canal opening and sealed to the side of the head with Thomas Lubriseal grease. Sound was transduced by a Beyer headphone coupled to the delivery tube. Pure tones were generated by a Wavetek model 184 waveform generator, amplified, passed through a Coulbourn electronic switch, and attenuated. Stimuli were 50 -msec-duration tone bursts with $5 \mathrm{msec}$ rise/fall times and were presented at a rate of two bursts/second.

The sound pressure level at the ear canal opening was measured with a Bruel and Kjaer half-inch condenser microphone connected to a calibrated probe tube inserted down the center of the sound delivery tube. Prior to each experiment the system was calibrated with a Bruel and Kjaer type 2112 Audio Frequency Spectrometer.

Electrophysiology. Recordings of neuron unit clusters were made with glass-insulated tungsten microelectrodes with exposed tips of approximately $40 \mu \mathrm{m}$. The electrical activity at the electrode tip was amplified, bandpass filtered $(0.5-10 \mathrm{kHz})$, viewed on an oscilloscope, and monitored over a loudspeaker.

All recordings were carried out inside a sound-attenuated IAC chamber. The microelectrode was mounted on a remotely controlled Burleigh

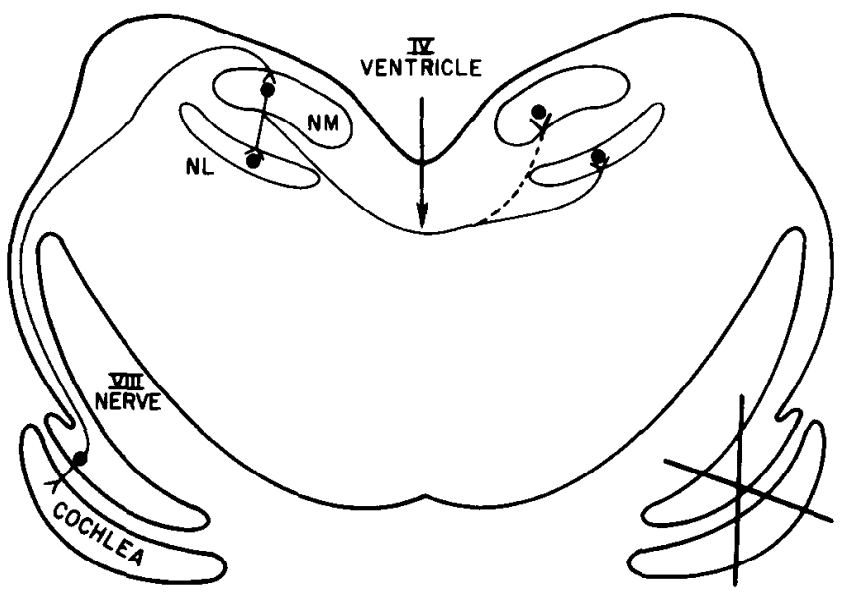

Figure 1. Schematic drawing of a transverse section through the chick brainstem showing the normal connections of the auditory pathway and the connections following early otocyst removal. The auditory nerve normally projects to the ipsilateral NM, which in turn sends a bilateral projection to NL. Following unilateral extirpation of the otocyst, the bilateral projection from NM to NL is maintained and the contralaterally travcling NM axon sprouts a branch (broken line) that innervates the "deafferented" NM on the operated side of the brain. The branch arises near the medial border of the NL on the operated side of the brain. (From Jackson and Parks, 1988, their Fig 1.)

Inchworm microdrive and advanced through the cerebellum and into the underlying brainstem. Entry of the electrode into NM was indicated by a characteristic pattern of rhythmic spontaneous multiunit firing and large-amplitude evoked multiunit activity. The approximate boundaries of NM were first determined with three to five electrode penetrations. Then, two punctures separated by approximately $500 \mu \mathrm{m}$ were made along the anteromedial-posterolateral dimension of the nucleus. In each puncture, characteristic frequency (CF) was measured at $100 \mu \mathrm{m}$ intervals. The $C F$ was determined using audiovisual criteria and was defined as the frequency at which the unit cluster had its lowest excitatory response threshold. At the termination of each puncture, one or two small marking lesions were made at different depths along the track by passing a $10 \mu \mathrm{A}$ current for $5 \mathrm{sec}$ through the tip of the electrode. The marking lesions were later used to calculate tissue shrinkage and determine the location of the unit clusters.

Histology. At the termination of the experiment, the embryo was killed with sodium pentobarbital $(50 \mathrm{mg}$ Nembutal, i.p.; Abbott Laboratory). The embryo was then staged by measuring the length of the third toe (Hamburger and Hamilton, 1951). The embryonic ages referred to in this study are the number of days of incubation corresponding to the Hamilton and Hamburger stages. The head was blocked in the plane of the electrode tracks and immersed in Bouin's fixative for $24 \mathrm{hr}$.

The absence of the cochlea and cochlear nerve on the side of otocyst removal was confirmed through careful dissection of the temporal bone. Only embryos in which the cochlea and cochlear nerve were completely absent were included in the study.

Each brainstem was embedded in paraffin. Serial $10 \mu \mathrm{m}$ coronal sections were cut through the region of the brainstem that included NM and were stained with cresyl violet.

Data analysis. Data analysis followed a protocol similar to that used previously (Rubel and Parks, 1975; Lippe and Rubel, 1985). Electrode tracks were identified by examining the stained tissue sections under a light microscope. Tracks that passed through NM were reconstructed on outline drawings of coronal sections through the nucleus. The marking lesions and calculated tissue shrinkage were used to plot the locations of all unit clusters on the reconstructed tracks. Only unit clusters within NM were used in analyses.

Each unit cluster's percentile position along the posterior-to-anterior (\%P-A) and lateral-to-medial (\%L-M) dimensions of the nucleus was calculated by methods described previously (Lippe and Rubel, 1985). The dorsal-ventral dimension of NM represents isofrequency columns (Rubel and Parks, 1975; Lippe and Rubel, 1985). Conventional single and multiple linear regression methods were used to correlate charac- 


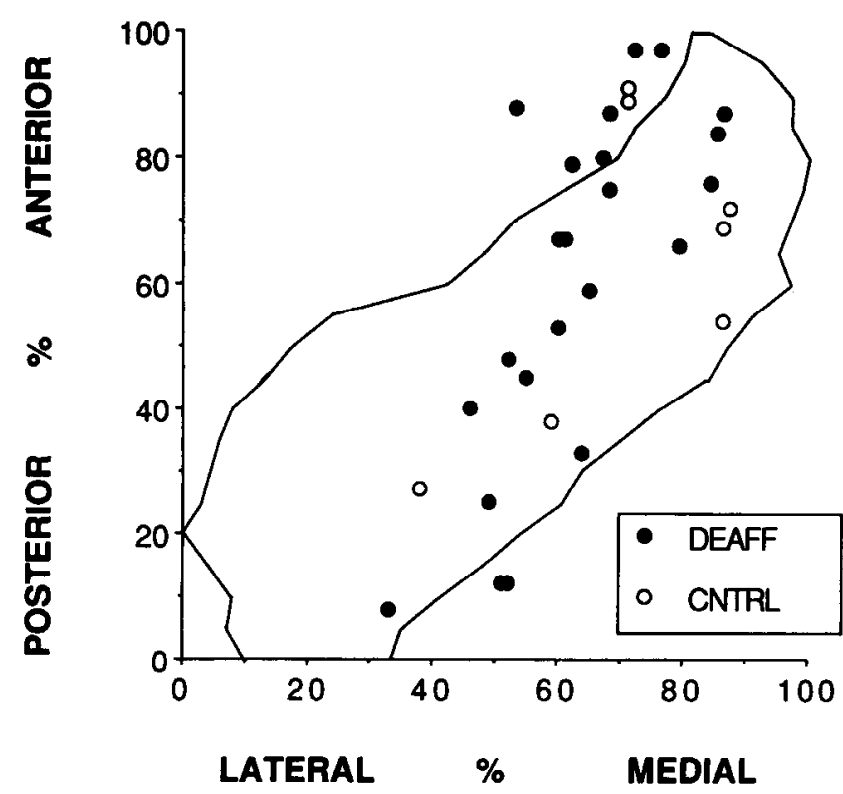

Figure 2. The positions of all unit clusters recorded in operated embryos are shown on a mean horizontal projection of NM (projection taken from Lippe and Rubel, 1985). Deafferented $(D E A F F)$ and control (CNTRL) refer, respectively, to NM ipsi- and contralateral to the side of otocyst removal. Small differences in sectioning angle among brains account for the fact that some units lie outside the boundaries of the mean horizontal projection.

teristic frequency with position in the nucleus. Statistical comparisons of characteristic frequency were performed using unpaired two-tailed $t$ tests and the Fisher exact probability test (Siegel, 1956).

\section{Results}

Surgical procedures on avian embryos greatly increase embryonic mortality (Parks, 1979). Forty-five of the approximately 450 embryos that underwent otocyst removal survived to $20 \mathrm{~d}$ of incubation. Embryos that had broken into the air space, that showed deformities in the development of the head, or whose physiological condition deteriorated while being prepared for recording were not studied. The 29 embryos used in electrophysiological mapping experiments yielded a total of $35 \mathrm{mul}-$ tiunit clusters within the boundaries of NM: 28 clusters in the right NM (deafferented), ipsilateral to otocyst removal, and 7 in the left NM (control). The age of all embryos at death corresponded to E17-E18 in the Hamilton and Hamburger (1951) reference series, although their chronological age was E20. Fiftysix unit clusters recorded previously from unoperated E16-E17 embryos provided normative data on tonotopic organization at this developmental age (Lippe and Rubel, 1985; Lippe, 1987).

\section{General observations: anatomy and electrophysiology}

Careful dissection confirmed that the cochlea and cochlear nerve were absent on the side of otocyst extirpation in all embryos studied electrophysiologically. The overall shape of NM on the two sides of the brain appeared similar. In some embryos the spatial relationship of NM to NL was altered on the operated side such that NM was closer than normal to the medial edge of NL. In addition, the deafferented NM was noticeably smaller in volume than the control NM on the unoperated side of the brain. The difference in volume likely reflects decreases in neuronal size and number that result from otocyst extirpation (LeviMontalcini, 1949; Parks, 1979).
High levels of spontaneous and sound-evoked multiunit activity were recorded in the NM ipsilateral to otocyst removal. The multiunit activity appeared similar to that in the control NM and in the NM of unoperated embryos (Lippe and Rubel, 1985; Lippe, 1987). Sound stimulation produced an increase in unit firing, and response thresholds were within the normal range of approximately 65-85 dB (sound pressure level). Spontaneous discharges occurred in a rhythmic pattern similar to that described previously in the normal NM (Lippe, 1985; Lippe and Rubel, 1985). Spontaneous activity in the NM of normal embryos and hatchlings is eliminated following cochlea removal (Lippe, 1985; Born et al., 1991). Thus, the background firing in the deafferented NM most probably reflects activity that is generated in the left cochlea and transmitted to the contralateral side of the brain by the abnormal NM-to-NM projection.

\section{Tonotopic organization}

Figure 2 shows the recording locations on an average planar projection of NM of the 35 unit clusters recorded from operated embryos and used in the quantitative analysis of tonotopic organization. All regions of the deafferented NM, except the most posterolateral portion of the nucleus, were sampled. Most of the posterolateral area is innervated by the lagena rather than the basilar papilla and is unresponsive to the sound frequencies used here (>100 Hz; Boord and Rasmussen, 1963; Rubel and Parks, 1975; Lippe and Rubel, 1985). The seven unit clusters in the control NM were recorded at various locations along the posterolateral-to-anteromedial dimension of the nucleus. These represent a sampling of different frequency regions along the tonotopic axis of the nucleus (see Fig. 5).

The tonotopic organization of the deafferented NM was qualitatively similar to that observed previously in unoperated embryos (Fig. 3). CF increased progressively as the recording electrode was moved from posterior to anterior (P-A) or lateral to medial (L-M) in the brain. Electrode penetrations that passed dorsoventrally through the nucleus encountered unit clusters whose CFs typically did not differ by more than $200 \mathrm{~Hz}$. Single linear regression analysis confirmed that $C F$ varied significantly along both the P-A $(r=0.89)$ and L-M $(r=0.81)$ dimensions of the nucleus ( $p$ values $<0.0001$ ). These correlations are similar to $r=0.82(p<0.0001)$ and $r=0.83(p<0.0001)$ for the P-A and L-M dimensions, respectively, in unoperated embryos.

Since CF varies along both positional dimensions, the relationship between frequency and location in NM can be best described with a multiple linear regression of CF on P-A and L-M position. The CF of neurons in NM ipsilateral to otocyst removal is predicted by the equation $\mathrm{CF}=12.7(\% \mathrm{P}-\mathrm{A})+$ $12.3(\% \mathrm{~L}-\mathrm{M})-351$. The comparable equation in unoperated embryos is $\mathrm{CF}=10.28(\% \mathrm{P}-\mathrm{A})+9.77(\% \mathrm{~L}-\mathrm{M})-256$. The multiple regression analysis allows three indices of tonotopic organization to be quantified and compared between normal and deafferented nuclei: (1) the amount of variation in CF that is attributable to position in the nucleus, (2) the orientation of the tonotopic gradient, and (3) the relationship between CF and position of cells in the nucleus.

The amount of variation in CF that is accounted for by location in the nucleus is equal to the coefficient of multiple determination for the multiple linear regression of CF on position $\left(r^{2}\right)$. This measure can also be termed the "predictability" of $\mathrm{CF}$, following the terminology of Parks et al. (1987). In normal embryos, position accounts for $75 \%$ of the variation of CF ( $r=$ $0.87 ; p<0.0001$ ). In the deafferented NM of operated embryos, 

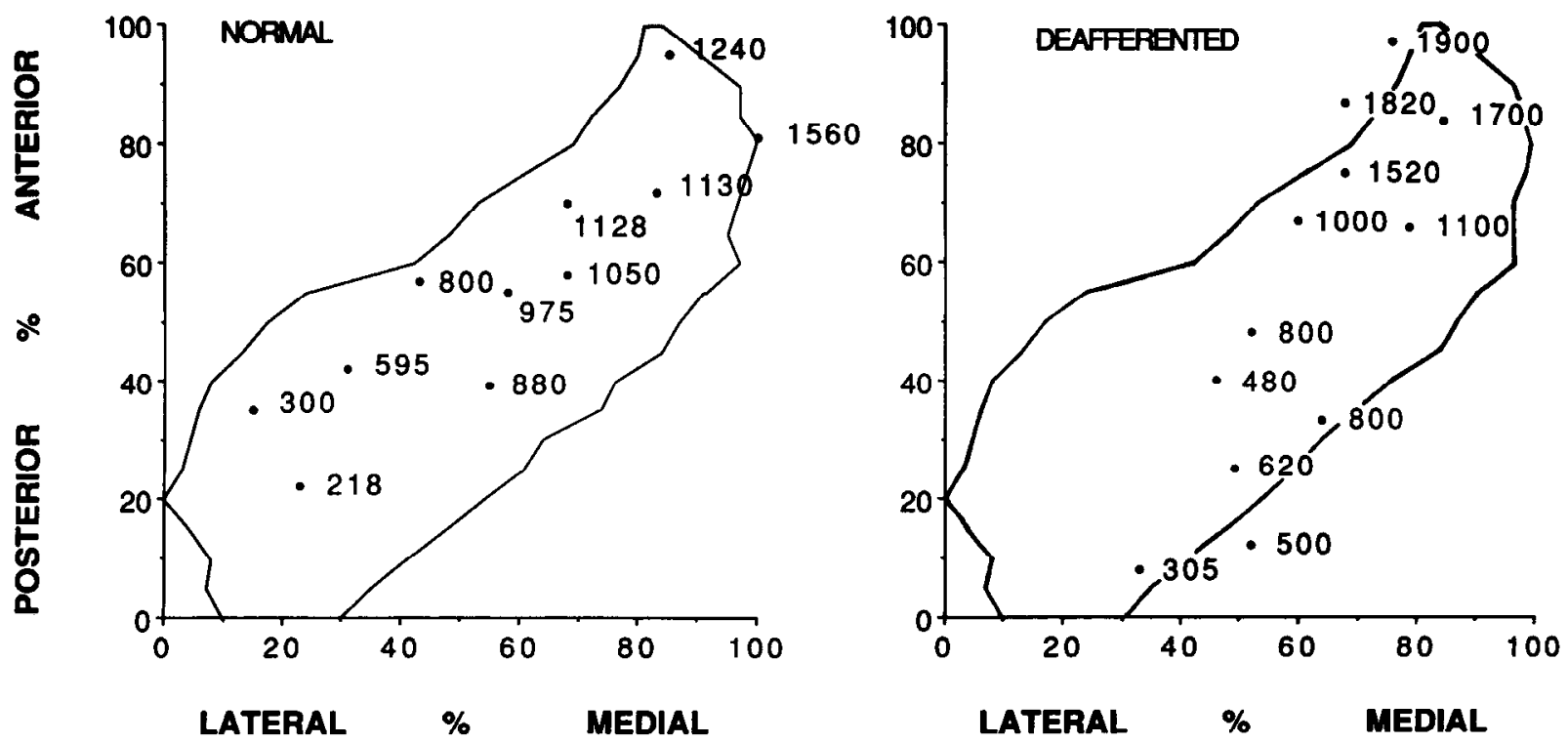

Figure 3. The positions and CFs (in Hz) of representative samples of unit clusters are shown on a mean horizontal projection of NM. Unit clusters are from several different embryos. Note that higher CFs are observed as the recording electrode is moved anteriorally or medially in the deafferented $\mathrm{NM}$ as well as in the NM of normal (unoperated) embryos.

$r^{2}=84 \%(r=0.91 ; p<0.0001)$. Thus, the "predictability" of CF in the deafferented NM is as great as that in the normal NM of unoperated embryos.

It was of particular interest to know if the orientation of the tonotopic gradient in the deafferented NM is also normal. A normal alignment in functional topography would imply that the aberrant NM-to-NM projection has the same topographic orientation in NM as does the normal auditory nerve projection to the nucleus. The orientation of the gradient is defined as the direction of increasing CF in the nucleus. The angle of the gradient with respect to the rostrocaudal and mediolateral axes of the brain is calculated from the ratio of the coefficients in the multiple linear regression equation (see Fig. 6 in Lippe and Rubel, 1985). Figure 4 shows that the orientation of the tonotopic gradient in the deafferented NM $\left(45.9^{\circ}\right)$ and normal NM $\left(46.5^{\circ}\right)$ is virtually identical. In both, the tonotopic gradient extends along the long axis of the two-dimensional planar projection. Low frequencies are located postcrolatcrally, and neu- rons responding to high frequencies are located progressively antcromedially. Neurons located along lines oriented orthogonal to the tonotopic axes constitute isofrequency bands. The small discrepancy of $0.5^{\circ}$ in the angle of the gradients is well within the approximately $5^{\circ}$ variation that may occur between different subgroups of normal embryos (W. R. Lippe, unpublished observations).

The quantitative relationship between $\mathrm{CF}$ and location along the tonotopic gradient in NM ipsi- and contralateral to otocyst removal and in normal embryos is shown in Figure 5. Examination of the scatter plots and single linear regression lines confirms what is indicated qualitatively in Figures 3 and 4: progressively higher CFs are observed as the recording electrode is moved anteromedially along the tonotopic axis in both the deafferented and the normal NM. The data also show that the CF at a given location along the tonotopic axis tends to be higher in the deafferented NM than in the NM of unoperated embryos. The divergence of the regression lines indicates that the mag-

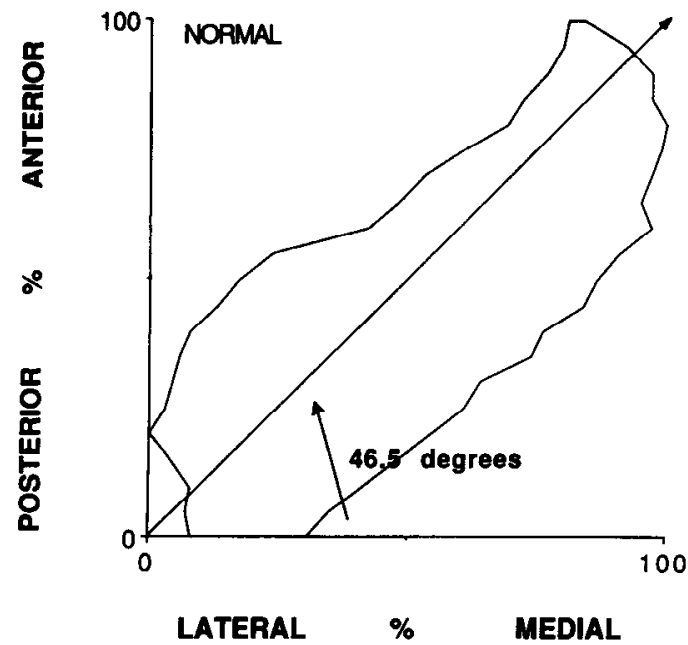

LATERAL

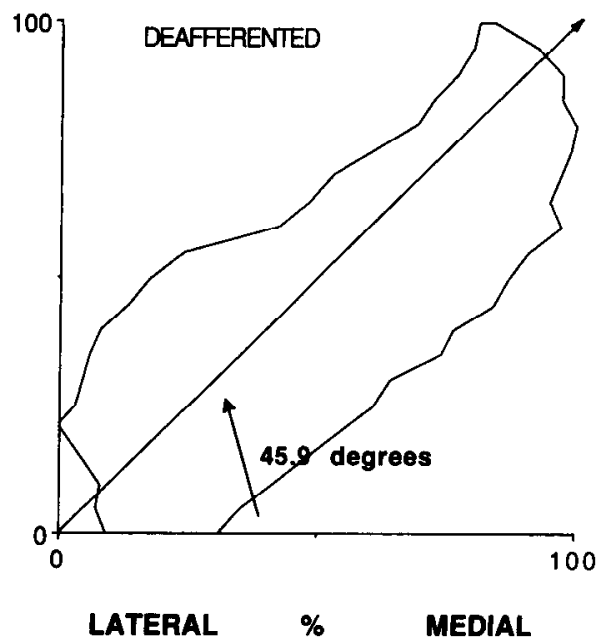

Figure 4. The orientation of the tonotopic axis in the normal and deafferented NM is represented on a mean horizontal projection of the nucleus. The arrow points in the direction of increasing $\mathrm{CF}$. The angle of the tonotopic axis with respect to medial-lateral axis is indicated. 


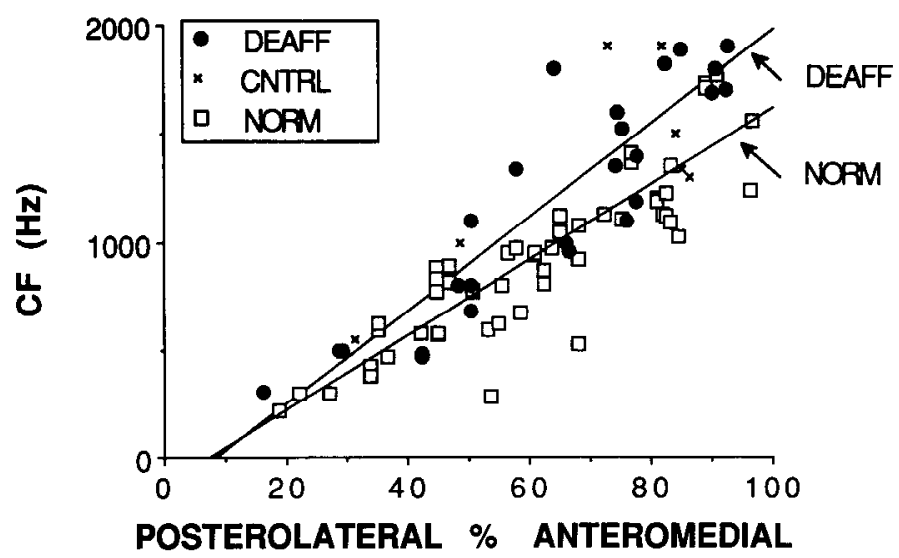

Figure 5. Comparison of the tonotopic organization: scatter plots and best-fitting linear regression lines relating $C F$ to percentage distance along the tonotopic gradient of NM. NORM, normal unoperated embryos (data from Lippe and Rubel, 1985; Lippe, 1987); $D E A F F$, ipsilateral to otocyst removal; $C N T R L$, contralateral to otocyst removal.

nitude of the difference in frequency between the deafferented and normal NM is greater at more anteromedial (higher-frequency) locations in the nucleus.

While examination of Figure 5 suggests that deafferented NM units respond optimally to higher frequencies than normal NM units only at high-frequency regions (anteromedial locations) in the nucleus, further analysis reveals an overall difference. Figure 6 shows the difference between the CFs observed in the deafferented NM and the frequencies predicted by the regression formula for our normal embryos, when this difference is measured in octaves rather than absolute hertz. This analysis reveals that the proportional difference in CF between the deafferented and normal NM does not vary reliably along the tonotopic gradient $(r=0.09 ; p>0.05)$.

Figure 5 also shows that the relationship between $C F$ and position in the nucleus is shifted toward higher frequencies in the control as well as in the deafferented NM of operated embryos. Both the proportion of units with CFs higher than the values predicted by the regression equation from unoperated embryos $(5 / 7,71 \%$, vs. $21 / 28,75 \% ; p=0.59)$ and the mean difference $( \pm$ SD) between observed and predicted frequencies $(0.26 \pm 0.32$ vs. $0.31 \pm 0.30$ octaves; $l=0.34 ; p>0.05)$ are similar in the control and deafferented NM. These findings indicate that the shift in tonotopicity toward higher frequencies does not result from the aberrant NM-to-NM projection.

\section{Discussion}

The tonotopic organization of NM in normal embryos reflects the systematic representation of frequency along the basilar papilla and the orderly topographic projection of the auditory nerve onto NM. Unilateral otocyst removal during early embryonic development prevents development of the ipsilateral cochlea and cochlear nerve and induces the formation of a functional projection from the contralateral NM to the NM on the operated side of the brain (Jackson and Parks, 1988). The present results show that the anomalous projection establishes a tonotopic organization in the deafferented $\mathrm{NM}$, and that the spatial organization of the tonotopicity is essentially identical to that in normal embryos. A gradient of increasing CF extends from posterolateral to anteromedial in the nucleus, and the orientation of the tonotopic gradient is normal.

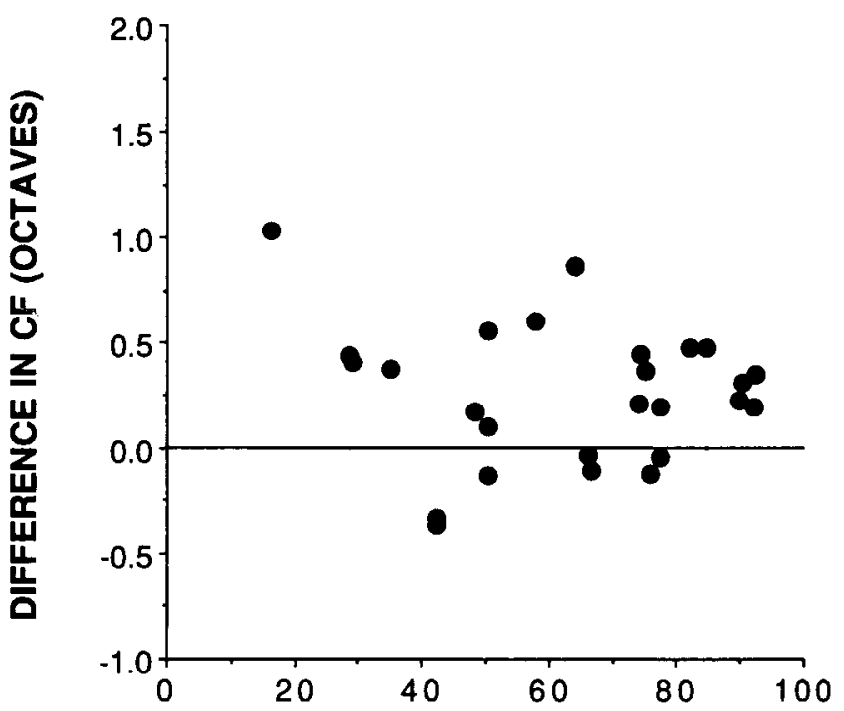

POSTEROLATERAL \% ANTEROMEDIAL

Figure 6. The difference (in octaves) between the CF observed for each unit cluster in the deafferented NM and the CF predicted by the regression equation for normal embryos is plotted as a function of percentage distance along the tonotopic gradient. $\mathrm{CF}_{\text {predicted }}=17.5(\% \mathrm{P}-\mathrm{A})$ -136 . Difference (in octaves) $=\log _{2}$ (observed CF/predicted CF).

\section{Formation of topographic maps by abnormal projections in other systems}

Several other examples of abnormal projections establishing normal topographic maps are described in the literature. In the majority of these instances, the anomalous projections form orderly maps in target structures to which their axonal populations normally project. For example, supernumerary eyes and cyes transplanted to ectopic locations establish normally ordered retinotectal projections (Law and Constantine-Paton, 1981; Harris, 1982). In Siamese cats a number of retinal afferents are misrouted in the optic chiasm and project to the contra- rather than the ipsilateral lateral geniculate nucleus. The aberrant retinogeniculate fibers, although specified incorrectly with respect to side of the brain, form a normally ordered retinotopic projection (Guillery and Kaas, 1971). The present finding is unusual in that, with the exception of the very small number of transient NM-NM projections present very early in some embryos, NM neurons do not normally project to the contralateral nucleus (Parks and Rubel, 1975; Young and Rubel, 1983, 1986). Thus, a topographic map was established in a completely novel target. An even more striking example of this is the finding that rerouting retinal afferents into the auditory pathway during early development results in the establishment of a retinotopic map in the auditory cortex (Frost, 1981; Pallas et al., 1990; Roe et al., 1990). However, in this latter example, unlike the present study, it is difficult to analyze the degree to which a topographically ordered map conforms to a "normal" orientation.

\section{Possible mechanisms underlying the development of tonotopicity in the deafferented NM}

The frequency organization of the deafferented NM undoubtedly has its basis in the anatomical pattern of the NM-to-NM projections. The projection arises as a vertically directed branch of the contralateral NM axon at the medial border of NL (see 
Fig. 1). Individual branches enter the deafferented NM ventrally and arborize over small areas of the nucleus (Jackson and Parks, 1988a). The present physiological results imply that the aberrant projection is topographically organized such that a given region in the NM contralateral to otocyst removal projects to the same relative location in the deafferented NM. Functional topography provides only an indirect assay of anatomical connections. Thus, the present results do not indicate how precisely the topography of axon terminals in the deafferented nucleus mirrors the topography of their parent cell bodies in the contralateral NM. Additionally, it is not known if the underlying pattern of axonal connections can alone account for the tonotopic map. Findings in mammals suggest that functional topography may be regulated, in part, by physiological processes that select a subset of inputs from a larger number of potential inputs that are available anatomically (Kaas et al., 1983; Wall et al., 1986; Robertson and Irvine, 1989; Roe et al., 1990). It is conceivable that similar mechanisms may operate in the deafferented NM and contribute to the generation and maintenance of tonotopicity. Examining the topography of thc abcrrant projection anatomically will help to assess this possibility.

Several mechanisms, including the spatial ordering of axons in sensory nerves, timing of axon outgrowth, substrate guidance, chemoaffinity, and electrical activity, have been invoked to explain the formation of topographically organized connections (Udin, 1988). The possibility that some of these mechanisms may contribute to the presumed topography of the aberrant projection can be evaluated in light of the known characteristics of the projection and the effect of otocyst removal on NM. In this context it is important to emphasize that order and orientation (polarity) are separate aspects of the tonotopic map. Thus, it is conceivable that the mechanism responsible for the ordering of the aberrant axon terminals into a tonotopic array may be different from the mechanism that determines the array's alignment with respect to the axes of the brain.

Order. The basis for the topographic ordering of the NM-toNM projection is unknown. However, it can be speculated that several potential mechanisms may contribute. One possibility is that the topographic map results, in part, from the maintenance of preexisting fiber order. The contralaterally projecting NM axons from' which the aberrant projection arises are ordered rostrocaudally at the level of NL. If the aberrant axons take the shortest possible route to the deafferented NM, possibly using the contralaterally projecting axons of the target NM neurons as a guide, then a rostrocaudal ordering would be maintained in the anomolous projection. While this is a very plausible mechanism, there are several reasons for believing that it alone cannot account for the mapping function observed. It does not explain the tonotopic order across the mediolateral dimension of the nucleus. Furthermore, developmental studies suggest that fiber order among contralaterally projecting NM axons does not itself account for the topographic specificity of this projection to NL (Young and Rubel, 1983).

A second possibility is that topographic ordering is achieved by a matching process wherein axon terminals bear positiondependent cytochemical markers matched with corresponding cues in the deafferented nucleus (Sperry, 1963). Two observations are relevant to this possibility. First, neuron number in the deafferented NM is reduced by approximately $30 \%$ (LeviMontalcini, 1949; Parks, 1979). The present experiment is thus similar in design and outcome to size mismatch studies in the visual system that have reported formation of normally ordered retinotopic maps in the tectum and superior colliculus following surgical reduction in the size of these structures (Gaze and Sharma, 1970; Yoon, 1971; Finlay et al., 1979). The present results are consistent with these findings and suggest that any matching that might occur does not work on the basis of highly specific cell-to-cell affinities. Axon terminals must exhibit a graded rather than an absolute preference for postsynaptic positional markers in NM. Second, since the NM ipsilateral to otocyst removal develops in the absence of its normal cochlear innervation, postsynaptic positional cues in NM are most probably intrinsic to the nucleus and not transmitted from the cochlear epithelium.

A final possibility is that the aberrant projection is self-organizing. Mutual interactions among axons in which relative position is coded by chemical labels or the pattern of action potential discharge would allow topographically ordered connections to be formed on an informationless target structure (Willshaw and von der Malsburg, 1976). Several lines of evidence indicate that action potential discharge, particularly the relative synchrony of firing among presynaptic axons, can contribute to the formation or refinement of topographically ordered connections (Meyer, 1983; Schmidt and Edwards, 1983; Fawcett and O'Leary, 1985; Reh and Constantine-Paton, 1985; Sanes and Constantine-Paton, 1985; Dubin et al., 1986; Stryker, 1986). In this context it is interesting that we observed a synchronous pattern of spontaneous firing in the deafferented NM. Neurons in close spatial proximity to each other discharged synchronously at periodic intervals. This rhythmic pattern of synchronous spontaneous discharge is identical to the patterned spontaneous firing that occurs in NM and NL of normal embryos (Lippe, 1985; Lippe and Rubel, 1985). Aberrant axons are present in the deafferented NM as early as E7 (Parks and Jackson, 1986). The development of the NM-to-NM projection has not been studied in detail. However, during normal embryonic development the afferents to NM and NL undergo a period of reorganization that entails the elimination of transient terminals or a shifting of axonal connections (Jackson and Parks, 1982; Young and Rubel, 1986). These events occur during and subsequent to E11-E12, the time of onset of functional synaptogenesis in NM and NL (Jackson et al., 1982). It is tempting to speculate that patterned spontaneous activity may guide these changes or in some other manner contribute to the development of patterned connections, in the deafferented NM as well as in NM and NL of normal embryos. This idea can be tested experimentally by examining the pattern of the induced NM-toNM as well as the normal NM-to-NL projections following the elimination of all action potential discharge by bilateral otocyst removal.

Orientation. The orientation of the tonotopic axis in NM arises from the alignment of tonotopically ordered arrays of axonal projections within the nucleus. The observation that orientation of the tonotopic axis in the normal and deafferented NM is virtually identical strongly implies that NM contains intrinsic postsynaptic alignment cues that emerge independently during development and entrain the tonotopic map to a proper orientation. The alternative possibility, that alignment information is impressed upon the nucleus by its afferents, is unlikely. If this were true, then one would expect that alignment information in the aberrant branches and their parent axons would be similar. Consequently, the orientation of the tonotopic axis in the deafferented NM and the NL on the operated side of the brain would be the same. The orientation of the tonotopic axis in NM and NL differs by approximately $20^{\circ}$ in normal embryos 
(Lippe and Rubel, 1985). The tonotopic organization of the laminar nucleus was not analyzed quantitatively in the present study. However, recordings were routinely made from the NL ipsilateral to otocyst removal, and these provided no indication that the tonotopicity of the nucleus differed from normal.

The idea that orientation is determined intrinsically is also supported by observations on the effect of otocyst removal on morphological gradients in NM and NL. Several developmental events in NM or NL occur in a direction that parallels the tonotopic axis in the respective nuclei. These include, for example, the elimination of transient dendritic processes in NM and changes of dendritic length in NL (Smith, 1981; Parks and Jackson, 1984; Young and Rubel, 1986). Parks has observed that both these processes are unaffected by otocyst removal (Parks and Jackson, 1984; Parks et al., 1987). The fact that these morphological transformations occur along spatial gradients virtually identical to the tonotopic axis and proceed normally in the absence of cochlear innervation has led Parks to conclude that "each brainstem auditory nucleus is organized along an intrinsically determined axis which specifies how incoming afferent axons will be organized" (Parks et al., 1987). This conclusion and the present findings are similar to observations indicating that central visual nuclei contain inherent topographical polarity cues that develop independently of the peripheral sensory epithelium (Kaiserman-Abramof et al., 1980; ConstantinePaton and Ferrari-Eastman, 1981).

The present findings indicate that the induced branches, although arising from parent axons that innervate NL, are nonetheless capable of "reading" orientation cues intrinsic to NM. Looked at from a somewhat different perspective, the results show that orientation cues intrinsic to NM are equally accessible to and can be read by a "foreign" population of axons as well as by the cochlear afferents that normally innervate the nucleus. It is possible that the cues for tonotopic orientation are different in NM and NL, and that NM axons are capable of reading both types of cues. However, a more parsimonious explanation is that the cues for tonotopic orientation are more ubiquitous than previously suspected. That is, it is likely that orientation cues are similar in NM and NL and that the induced branches are reading cues comparable to those that their parent $\mathrm{NM}$ axons normally encounter in their projection to NL.

\section{Discrepancy in $C F$}

The only noticeable discrepancy in tonotopicity is that CF at a given location along the tonotopic gradient was higher in the deafferented NM than in the NM of unoperated embryos. It is unlikely that this difference is related to the NM-to-NM projection since CFs in the NM contralateral to otocyst removal were also higher than normal. It is conceivable that early otocyst removal alters, through some unknown mechanism, the normal development of frequency representation along the contralateral basilar papilla or the papilla's projection onto NM, with the consequence that neurons in the control NM are stimulated by frequencies higher than normal. Interesting in this respect is the recent report showing that unilateral cochlea removal can affect the contralateral NM (Code et al., 1990). The shift toward higher frequencies would then be mirrored in the deafferented $\mathrm{NM}$ as a result of the NM-to-NM projection.

Although such a direct affect of otocyst removal cannot be excluded by the present results, it is much more probable that the discrepancy in CFs reflects age-related differences in tonotopicity between the operated and unoperated embryos. Neu- rons at a given location in NM become tuned to progressively higher frequencies during development (Lippe and Rubel, 1985; Lippe, 1987). The progressive increase in CF likely results from maturational changes in the place code along the basilar papilla (Rubel and Ryals, 1983; Lippe and Rubel, 1985; Lippe et al., 1986). Because embryos that had undergone otocyst removal were slightly older (staged to E17-E18) than unoperated controls (E16-E17), CFs would be expected to be higher in the operated embryos. Additionally, it is possible that the basilar papilla in embryos that had undergone otocyst removal was more mature at the time of recording than would be presumed from the embryo's developmental age, according to the Hamburger and Hamilton (1951) series. This would further contribute to higher CFs being observed in operated embryos. Developmental age is assessed by measurements of external bodily characteristics, such as beak and toe length. Whereas the developmental age of normal embryos corresponds to their total duration of incubation, the developmental age of operated embryos (E17-E18) was less than their age measured chronologically ( $20 \mathrm{~d}$ of incubation). Embryos that undergo otocyst removal typically exhibit a 2-3 d delay in gross structural development (Parks, 1978). However, it is quite possible that the development of the basilar papilla and the resulting increases of CF in NM are retarded to a lesser extent than is the development of external bodily characteristics.

\section{References}

Boord RL, Rasmussen GL (1963) Projection of the cochlear and lagenar nerves on the cochlear nuclei of the pigeon. J Comp Neurol 120:463-475.

Born DE, Durham D, Rubel EW (1991) Afferent influences on brainstem auditory nuclei of the chick: nucleus magnocellularis neuronal activity following cochlea removal. Brain Res 557:37-47.

Code RA, Durham D, Rubel EW (1990) Effects of cochlea removal on GABAergic terminals in nucleus magnocellularis of the chicken. J Comp Neurol 301:643-654.

Constantine-Paton M, Ferrari-Eastman P (1981) Topographic and morphometric effects of bilateral embryonic eye removal on the optic tectum and nucleus isthmus of the leopard frog. J Comp Neurol 196: 645-661.

Dubin MW, Stark LA, Archer SM (1986) A role for action-potential activity in the development of neuronal connections in the kitten retinogeniculate pathway. J Neurosci 6:1021-1036.

Fawcett JW, O'Leary DM (1985) The role of electrical activity in the formation of topographic maps in the nervous system. Trends Neurosci 8:201-206.

Finlay BL, Schneps SE, Schneider GE (1979) Orderly compression of the retinotectal projection following partial tectal ablation in the newborn hamster. Nature 280:153-155.

Frost DO (1981) Orderly anomalous retinal projections to the medial geniculate, ventrobasal and lateral posterior nuclei of the hamster. J Comp Neurol 203:227-256.

Gaze RM, Sharma SC (1970) Axial differences in the reinnervation of the goldfish optic tectum by regenerating optic nerve fibers. Exp Brain Res 10:171-181.

Guillery RW, Kaas JH (1971) A study of normal and congenitally abnormal retinogeniculate projections in cats. J Comp Neurol 143: 73-100.

Hamburger V, Hamilton $H$ (1951) A series of normal functional stages in the development of the chick embryo. J Morphol 88:49-92.

Harris WA (1982) The transplantation of eyes to genetically eyeless salamanders: visual projections and somatosensory interactions. $J$ Neurosci 2:339-353.

Jackson H, Parks TN (1982) Functional synapse elimination in the developing avian cochlear nucleus with simultaneous reduction in cochlear nerve axons branching. J Neurosci 2:1736-1743.

Jackson H, Parks TN (1988) Induction of aberrant functional afferents to the chick cochlear nucleus. J Comp Neurol 271:106-114. 
Jackson H, Hackett JT, Rubel EW (1982) Organization and development of brain stem auditory nuclei in the chick: ontogeny of postsynaptic responses. J Comp Neurol 210:80-86.

Kaas JHM, Merzenich MM, Killackey HP (1983) The reorganization of somatosensory cortex following peripheral nerve damage in adult and developing mammals. Annu Rev Neurosci 6:325-356.

Kaisermann-Abramof IR, Graybiel AM, Nauta WJH (1980) The thalamic projection to cortical area 17 in a congenitally anopthalmic mouse strain. Neuroscience 5:41-52.

Law MI, Constantine-Paton M (1981) Anatomy and physiology of experimentally produced striped tecta. J Neurosci 1:741-759.

Levi-Montalcini R (1949) Development of the acoustico-vestibular centers in the chick embryo in the absence of the afferent root fibers and of descending fibers tracts. J Comp Neurol 91:209-242.

Lippe WR (1985) Developmental changes in the pattern of spontaneous activity in the avian auditory system. Assoc Res Otolaryngol Abstr 9:176.

Lippe WR (1987) Shift of tonotopic organization in brain stem auditory nuclei of the chicken during late embryonic development. Hear Res 25:205-208.

Lippe WR, Rubel EW (1985) Ontogeny of tonotopic organization of brain stem auditory nuclei in the chicken: implications for development of the place principle. J Comp Neurol 237:273-289.

Lippe WR, Ryals BM, Rubel EW (1986) Development of the place principle. In: Advances in neural and behavioral development, Vol II (Aslin RN, ed), pp 155-203. Norwood, NJ: Ablex.

Meyer RL (1983) Tetrodotoxin inhibits the formation of refined retinotopography in goldfish. Develop Brain Res 6:293-298.

Pallas SL, Roe AW, Sur M (1990) Visual projections induced into the auditory pathway of ferrets. I. Novel inputs to primary auditory cortex (AI) from LP/pulvinar complex and the topography of the MGN-AI projection. J Comp Neurol 298:50-68.

Parks TN (1978) Afferent influences on the development of the avian brainstem auditory nuclei. $\mathrm{PhD}$ thesis, Yale University.

Parks TN (1979) Afferent influences on the development of the brain stem auditory nuclei of the chicken: otocyst ablation. J Comp Neurol 183:665-678.

Parks TN, Jackson H (1984) A developmental gradient of dendritic loss in the avian cochlear nucleus occurring independently of primary afferents. J Comp Neurol 227:459-466.

Parks TN, Jackson H (1986) Early destruction of the ear induces formation of a new functional brain stem auditory pathway. In: The biology of change in otolaryngology (Ruben RJ, Van De Water TR, Rubel EW, eds), pp 225-234. Amsterdam: Elsevier.

Parks TN, Rubel EW (1975) Organization and development of brainstem auditory nuclei of the chicken: organization of projections from n. magnocellularis to $\mathrm{n}$. laminaris. J Comp Neurol 164:435-448.

Parks TN, Rubel EW (1978) Organization and development of brain stem auditory nuclei of the chicken: primary afferent projections. J Comp Neurol 180:439-448.

Parks TN, Gill SS, Jackson H (1987) Experience-independent devel- opment of dendritic organization in the avian nucleus laminaris. $\mathbf{J}$ Comp Neurol 260:312-319.

Reh TA, Constantine-Paton M (1985) Eye-specific segregation requires neural activity in three-eyed Rana pipiens. J Neurosci 5:11321143.

Robertson D, Irvine DRF (1989) Plasticity of frequency organization in auditory cortex of guinea pigs with partial unilateral deafness. $J$ Comp Neurol 282:456-471.

Roe AW, Pallas SL, Jong-On H, Sur M (1990) A map of visual space induced in primary auditory cortex. Science 250:818-820.

Rubel EW, Parks TN (1975) Organization and development of brain stem auditory nuclei of the chicken: tonotopic organization of $n$. magnocellularis and n. laminaris. J Comp Neurol 164:411-434.

Rubel EW, Ryals BM (1983) Development of the place principle: acoustic trauma. Science 219:512-514.

Sanes DH, Constantine-Paton M (1985) The sharpening of frequency tuning curves requires patterned activity during development in the mouse, Mus musculus. J Neurosci 5:1152-1166.

Schmidt JT, Edwards DL (1983) Activity sharpens the map during the regeneration of the retinotectal projection in goldfish. Brain Res 269:29-39.

Siegel S (1956) Nonparametric statistics for the behavioral sciences. New York: McGraw Hill.

Smith ZDJ (1981) Organization and development of the brain stem auditory nuclei of the chicken: dendritic development in $\mathbf{n}$. laminaris. J Comp Neurol 203:309-333.

Sperry R (1963) Chemoaffinity in the orderly growth of nerve fiber patterns and connections. Proc Natl Acad Sci USA 50:703-710.

Stryker MP (1986) The role of neural activity in rearranging connections in the central visual system. In: The biology of change in otolaryngology (Ruben RJ, Van De Water TR, Rubel EW, eds), pp 211224. Amsterdam: Elsevier.

Udin SB (1988) Formation of topographic maps. Annu Rev Neurosci 11:289-327.

Wall JT, Kaas JH, Sur M, Nelson RJ, Felleman DJ, Merzenich MM (1986) Functional reorganization in somatosensory cortical areas 3b and 1 of adult monkeys after median nerve repair: possible relationships to sensory recovery in humans. J Neurosci 6:218-233.

Willshaw DJ, von der Malsburg C (1976) How patterned neural connections can be set up by self-organization. Proc R Soc Lond [Biol] 287:203-243.

Yoon MG (1971) Reorganization of retinotectal projection following surgical operations on the optic tectum in goldfish. Exp Neurol 33: 395-411.

Young SR, Rubel EW (1983) Frequency-specific projections of individual neurons in chick brainstem auditory nuclei. J Neurosci 3:13731378.

Young SR, Rubel EW (1986) Embryogenesis of arborization pattern and topography of individual axons in $n$. laminaris of the chicken brain stem. J Comp Neurol 254:425-459. 